Young Researcher Award of The Electrochemical Society of Japan (Sano Award)

\title{
Precise Control of Nanoscale Interface for Efficient Electrochemical Reactions
}

\section{Hiro MINAMIMOTO*,§ (D) and Kei MURAKOSHI\$§ (D)}

\author{
Department of Chemistry, Faculty of Science, Hokkaido University, Kita 10, Nishi 8, Kita-ku, Sapporo, Hokkaido 060-0810, Japan \\ *Corresponding author: minamimoto@sci.hokudai.ac.jp
}

\section{ABSTRACT}

Control of the nanoscale interface leads to efficient electrochemical reactions and unique molecular behaviors. This paper reviews our recent investigations on understanding the unique property of the electrochemical reactions at nanostructured interfaces. We have focused on the control of chemical reactions in the vicinity of metal nanostructures to condense the energy perturbations of electrons, ions, and photons. We have established an electrochemical nanostructure control method to achieve ultimate energy condensation. In addition, we have revealed the unique molecular behaviors induced by the strong interaction between the target molecules and the nanostructured electrode, originating from the hybridization of the electronic states showing collective excitation modes especially under the light illumination. Furthermore, we have attempted to efficiently control the electrochemical reactions and observe the unique reaction selectivity on the nanostructured electrodes. Through these investigations, we have proposed advantages for the control of nanostructured interfaces to overcome the current limitation of electrochemical reactions.

(C) The Author(s) 2021. Published by ECSJ. This is an open access article distributed under the terms of the Creative Commons Attribution 4.0 License (CC BY, http://creativecommons.org/licenses/by/4.0/), which permits unrestricted reuse of the work in any medium provided the original work is properly cited. [DOI: 10.5796/electrochemistry.21-00080].

Keywords : Localized Surface Plasmon Resonance, Plasmon-induced Electron Transfer, Electrochemical Surface-enhanced Raman Scattering, Hydrogen Evolution Reaction

\section{Introduction}

At the nanoscale electrode interface, a unique molecular response or electron transfer process can often be triggered depending on its morphology. ${ }^{1,2}$ This is derived from the appearance of specific surface properties, such as the distinct face of the metal or a change in the active sites. ${ }^{3,4}$ Another reason is considered as the electric field concentration effect because it changes various factors, such as the charging layer, resulting in enhancement of the currents, diffusion processes, or efficiency. ${ }^{5,6}$ Thus, accurate control of the interface structure is important for the electrochemical or photoelectrochemical reactions. In other words, through the precise control of the nanostructured interface, the unique reaction properties would be realized beyond the limitation of the system.

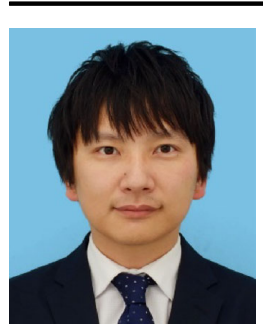

Hiro Minamimoto (Assistant Professor, Department of Chemistry, Faculty of Science, Hokkaido University)

Hiro Minamimoto was born in 1987. He received his Ph. D. Degree from the Department of Applied Chemistry at Osaka University in 2015. He is currently an assistant professor at Hokkaido University. He was awarded Young Researcher Award from The Electrochemical Society of Japan. His research interest is physical chemistry, especially electrochemical reactions at nanoscale interface and photoelectrochemistry. Hobby: Ski, Pro-wrestling, Music, and Drinking.

${ }^{\S}$ ECSJ Active Member

$\S \S$ ECSJ Fellow

H. Minamimoto (D) orcid.org/0000-0002-2360-577X

K. Murakoshi (D) orcid.org/0000-0003-4786-0115
Functionalization of the electrode is an important challenge in the electrochemistry field. One of the interesting features for the metal nanostructure could be defined as the localized surface plasmon resonance (LSPR) for photo-energy conversions. ${ }^{7-10}$ The LSPR, which is the collective oscillations of free electrons in the metal nanostructures induced by visible-light illumination, allows photon condensation near metal nanostructures beyond the light diffraction limit. Because the visible light accounts for $50 \%$ of sunlight, use of LSPR could be the promising approach for photochemistry and photoelectrochemical applications. Upon LSPR excitation, various unique phenomena can be triggered, such as the generation of a strongly localized photon energy field or electron-hole excitations. ${ }^{11,12}$ Because of these characteristics, LSPR not only enhances the reaction efficiency but also modulates the reaction pathways, which cannot be achieved via common visible-light illumination. ${ }^{13-15}$ Recently, plasmonic nanostructures have often been applied in photovoltaic devices because of their visible-light response ability. ${ }^{16}$ In addition to the applications in chemical reactions, plasmonic properties have been exploited in spectroscopy

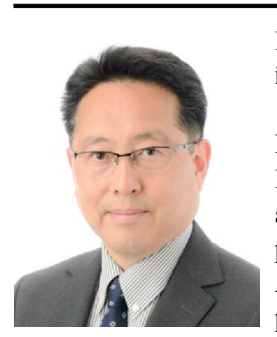

Kei Murakoshi (Professor, Department of Chemistry, Faculty of Science, Hokkaido University)

Kei Murakoshi was born in 1963. He received his B.Sc. in Science from Hokkaido University in 1986, and completed his Ph.D. in 1992. After postdoctoral positions at CNRS in Muedon, France, he joined as Assistant Professor at Osaka University in 1993, and promoted to Associate Professor in 1998. From 2003, he is current position at Hokkaido University. He was awarded the Association award from The Electrochemical Society of Japan in 2014. His research interests are electrochemical applications of nanostructures for energy conversions. Hobby: Exercise and Beer. 
measurements, e.g., the enhancement of Raman scattering or fluorescence photon emission. ${ }^{17-19}$ Thus, from both the electrochemical and plasmonic points of view, the control of the metal nanostructure interface could be recognized as an important issue to functionalize the electrode interface.

Because of the strong localization of the photon energy at the molecular scale, it is possible to observe unique molecular behavior at the nanostructure interface that originates from the interaction between the plasmonic field and molecules. For example, the huge intensity gradient can often break the electronic excitation selection rule. ${ }^{20-22}$ Recent theoretical and experimental studies have proposed the possibility of normally forbidden excitation processes at the plasmonic field. However, when the exciton and photon energies are close to each other, the coherent light energy exchanges between molecular excitons and the plasmon lead to the formation of a new energy hybridized state. This new energy state is called as the strong coupling regime and has the potential to change not only the absorption character but also the reaction pathway. ${ }^{23-25}$ Furthermore, because there is the strong field gradient force within the plasmonic field, the surface diffusion process can often be modulated, resulting in changes to the molecular condensation or reaction efficiency. ${ }^{26-28}$ These facts strongly suggest that the functionalized electrochemical reaction fields can be obtained through the control of the lightmatter interactions.

On the basis of the above background, we have investigated the electrochemical and photoelectrochemical reactions at the nanostructured surface via control of the metal nanostructures and/or electrochemical surface-enhanced Raman scattering (SERS) measurements. In the present account, we summarize our recent investigations. We have developed an accurate control method for nanostructures. Using this method, we have achieved ultimate light energy confinement in the sub-nanometer region. In addition, we have observed the unique surface process of molecules strongly coupled to the plasmonic field using electrochemical SERS measurements. Furthermore, we have examined the electron transfer process and the spatial distribution of the reaction active sites on the plasmonic photoconversion system via electrochemical SERS measurements. Finally, we introduce our recent approach for the control of the hydrogen evolution reactions using nanostructured electrodes with/without light illumination.

\section{Electrochemical Precise Control of Plasmonic Nanostructures}

It is well known that the plasmonic property strongly depends on not only the metal species but also its morphology, ${ }^{29-31}$ as indicated by the response of the electron cloud in the metal nanostructures to the electric field, described by the metal polarizability in the following equation: ${ }^{32}$

$$
\alpha(\lambda)=3 \varepsilon_{\text {med }} V_{N P} \frac{\varepsilon_{\text {metal }}(\lambda)-\varepsilon_{\text {med }}(\lambda)}{\varepsilon_{\text {metal }}(\lambda)+\chi \varepsilon_{\text {med }}(\lambda)}
$$

Here, $\lambda, \varepsilon_{\text {metal }}$, and $\varepsilon_{\text {med }}$ are the incident-light wavelength and the dielectric functions of the metal and the non-absorbing $\left(\operatorname{Im}\left[\varepsilon_{\text {med }}\right]=0\right)$ surrounding medium, respectively, and $V_{N P}$ and $\chi$ are the metal-nanoparticle volume and the geometrical factor, which depend on the metal species and geometry, respectively. Therefore, although it is still difficult for the conventional method, the accurate control of the metal nanostructure is quite important for efficient control of the plasmonic chemistry.

For separated dimer structures, unique photoresponse properties have been observed depending on the gap distance. ${ }^{33,34}$ Under the longitudinal polarization condition, the dipolar coupling recognized as the bonding dipolar plasmon (BDP) mode realizes strong light energy confinement. As the gap distance decreases down to the subnanometer-scale, the quantum tunneling effect occurs, resulting in the shielding BDP mode (shielded BDP (SBDP) mode). ${ }^{35}$ On the bridged dimer structure, not only the SBDP mode but also the charge transfer plasmon mode (CTP), which is derived from the oscillations of transferring electrons between the conductive interparticle junction, can be observed. ${ }^{35,36}$ It is interesting that the dimer structure with a gap distance of less than $1 \mathrm{~nm}$ allows for dramatic improvement of the light condensation efficiency via the excitation of the higher-order optically dark, but not completely forbidden, multipolar plasmon modes (e.g., the bonding quadrupolar plasmon (BQP) mode) ${ }^{37}$ Although the BQP mode has great advantages for various applications, because of technical difficulties, the flexible and highly reproducible excitation of BQP is quite difficult, especially under room-temperature conditions.

Until now, we have attempted to tune the electrochemical oxidation dissolution reaction at the $\mathrm{Au}$ nanostructure surface in aqueous halogen solution. ${ }^{38-40}$ As shown in Fig. 1, various dimer structures with different gap distances $(d)$ were prepared using electron beam lithography method. To dissolve the Au at the surface, the $\mathrm{Au}$ oxidation reactions $\left(\mathrm{Au}+4 \mathrm{Br}^{-} \rightarrow \mathrm{AuBr}_{4}^{-}+3 e^{-}\right)$were proceeded under the positive potential polarization condition. By precisely tuning the electrochemical potential and the halogen conditions, gradual changes in the gap distance were achieved while maintaining the shape of structures, as shown in Fig. 1. The optical property changes as a function of the potential polarization time were examined via electrochemical dark-field scattering spectroscopic measurements. From the scattering images in the figure, it was observed that the different tendency for the change in the scattering property was confirmed on the structure with $d=-5 \mathrm{~nm}$. On this structure, we observed strong scattering suppression at the specific potential polarization time. This scattering suppression indicates the excitation of the BQP mode by the formation of the sub-nanometer gap, leading to strong light energy condensation at the gap. According to the previous theoretical study, it was estimated that, ranging between -0.5 and $0.5 \mathrm{~nm}$, the CTP mode changes to the BQP mode, then the BDP mode is observed with increasing the gap distance more than $0.5 \mathrm{~nm} .{ }^{35,37}$ Therefore, it can be said that our electrochemical structure control method has achieved the precise tuning with the resolution of less than $1 \mathrm{~nm}$.

To demonstrate the advantage of our electrochemical structure control method, the electrochemical potential was switched during the oxidation dissolution process to stop the reaction. Figure 2 presents the time-series scattering spectra collected during the dissolution process on the dimer structure with $d=-5 \mathrm{~nm}$. The dissolution of the Au bridged dimer proceeded at $0.74 \mathrm{~V}$ vs. $\mathrm{Ag} /$ $\mathrm{AgCl}$ and was stopped by the potential scan to $0.6 \mathrm{~V}$ at $400 \mathrm{~s}$. The gradual peak shift indicated the change in the size of the conductive junction. Interestingly, the scattering suppression indicating the excitation of the BDP due to the sub-nm gap formation was kept for even $800 \mathrm{~s}$. This means that both the sub-nanometer gap and the higher-order modes were stable under ambient condition. Because the quadrupolar mode shows strong light energy condensation and relatively longer lifetime, our proposed electrochemical method can be recognized as innovative and useful for efficient light energy usage.

Recently, we have also applied our electrochemical structure control method to the two-dimensionally arranged metal nanoparticles (lattice structure) system. ${ }^{41}$ Within the lattice structure, the scattered light from one particle is absorbed by neighboring nanoparticles to excite the LSPR, resulting in the transformation from radiative to evanescent. This strongly coupled nanoparticles in the lattice structures excite the surface lattice resonance (SLR) mode. ${ }^{42-44}$ The optical properties of SLR mode is sensitively dependent on the LSPR properties, the distance between particles, and particle height because of the dipole interaction of respective particles. This SLR mode can effectively confine the light energy onto a wide two-dimensional region. Additionally, one of the 


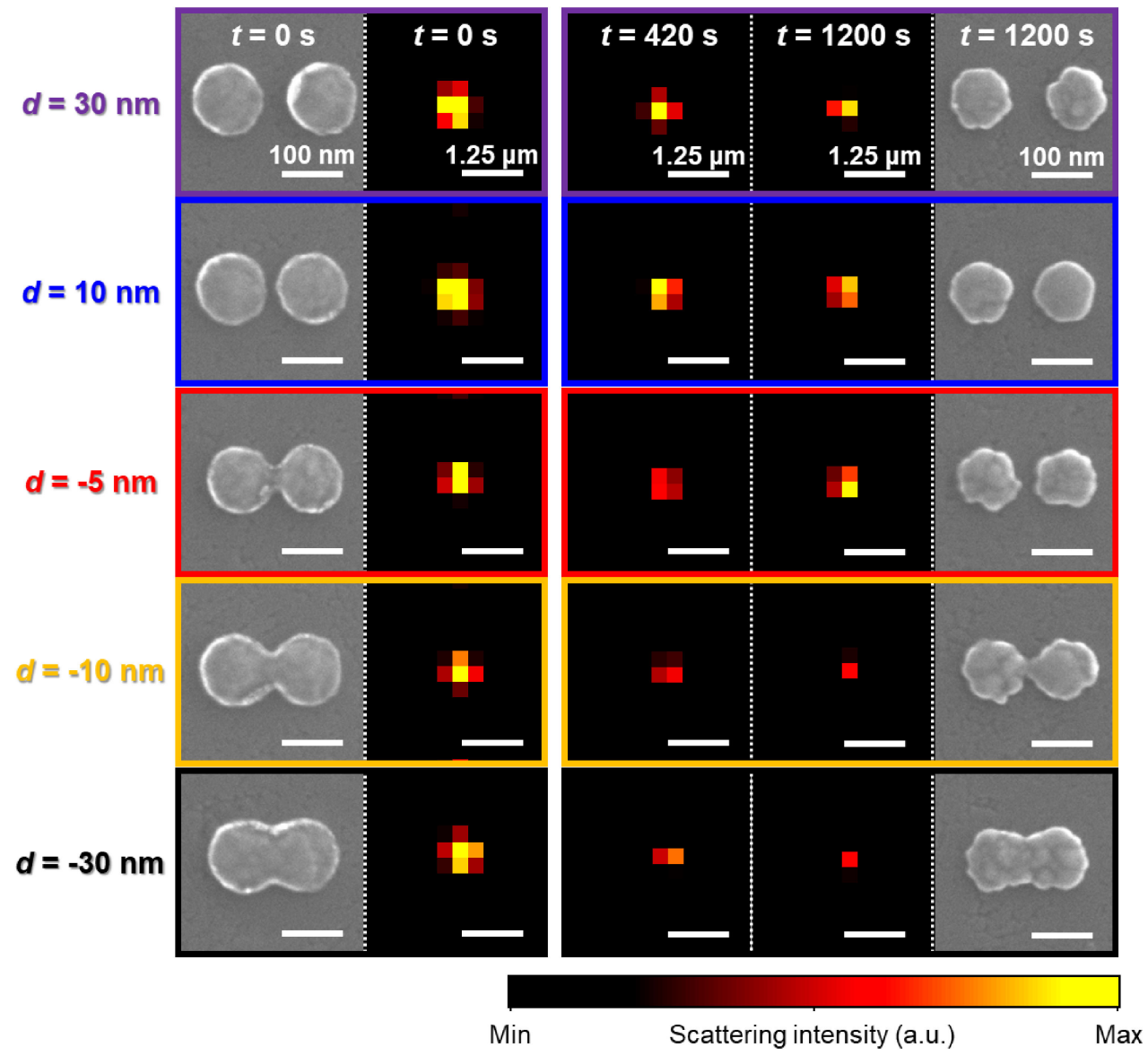

Figure 1. SEM and dark-field scattering images of Au nanodisk arrays with gap distances of $-30 \mathrm{~nm}$ (black), $-10 \mathrm{~nm}$ (orange), $-5 \mathrm{~nm}$ (red), $10 \mathrm{~nm}$ (blue), and $30 \mathrm{~nm}$ (violet) immersed in $10 \mathrm{mmol} \mathrm{dm}^{-3} \mathrm{KBr}$ aq. The applied potential was set at $0.74 \mathrm{~V}$ vs. $\mathrm{Ag} / \mathrm{AgCl}$. The potential polarization time for each case is indicated at the top of each part of the figure. Reprinted with permission from Ref. 40 .
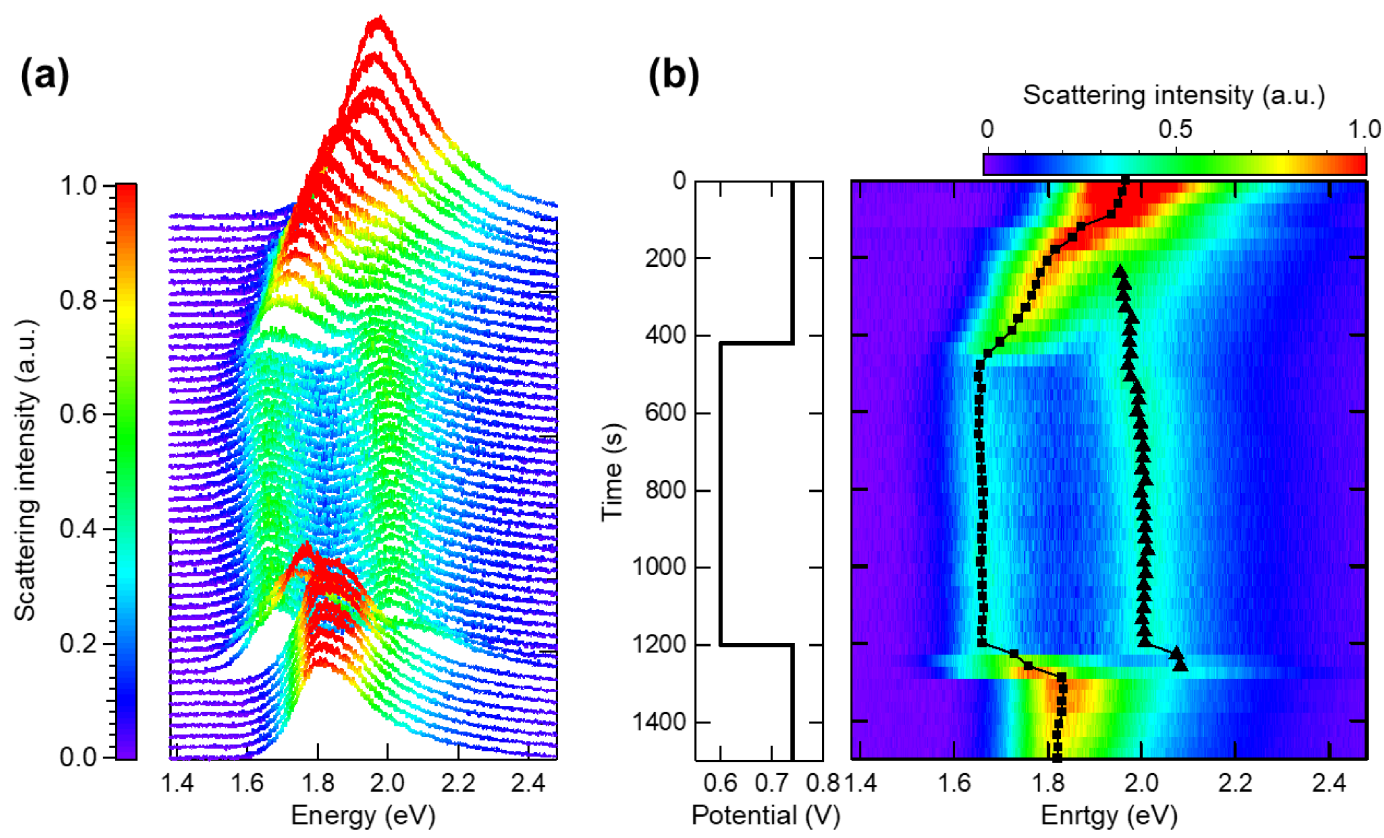

Figure 2. (a) Time series scattering spectra for bridged $\mathrm{Au}$ nanodisk arrays with a gap distance of $-5 \mathrm{~nm}$. The electrode potential was scanned from 0.74 to $0.6 \mathrm{~V}$ vs. $\mathrm{Ag} / \mathrm{AgCl}$ at $400 \mathrm{~s}$ and from 0.6 to $0.74 \mathrm{~V}$ vs. $\mathrm{Ag} / \mathrm{AgCl}$ at $1200 \mathrm{~s}$. (b) Image plot of the scattering intensity as a function of the potential polarization time, as estimated from (a). Reprinted with permission from Ref. 40.

important points for the SLR mode is that the lifetime of its mode is almost ten times longer than that for the LSPR mode (above $100 \mathrm{fs}) .{ }^{45}$ The dependence of SLR mode property on the distance between each particle (lattice spacing $=a$ ) can be found in Fig. 3a. When we applied our electrochemical structure control method to tune the optical property of the SLR mode, a gradual shift of the 
(a)
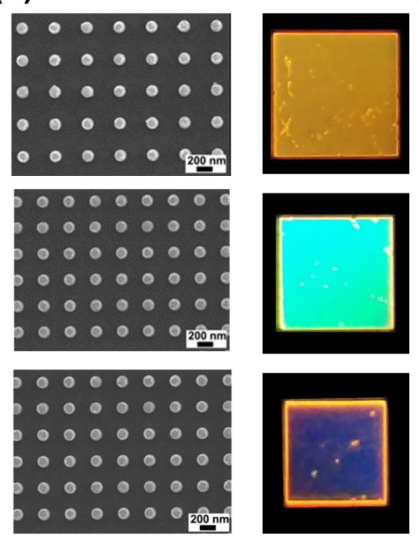

Energy / eV

(c)

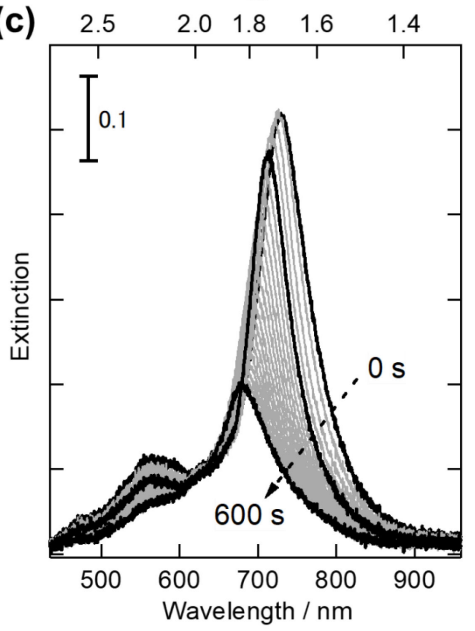

Energy / eV

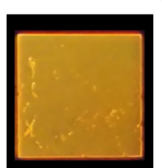

(b)

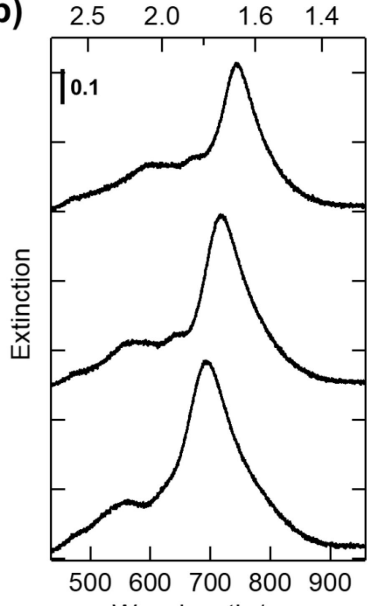

(d)
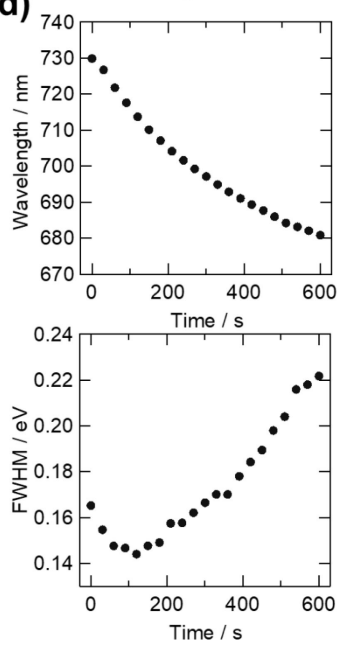

Figure 3. (a) SEM images and dark-field scattering images and (b) extinction spectra of Au lattices with $a=360$ (bottom), 400 (middle), and $440 \mathrm{~nm}$ (top) (the structure height and diameter of the disk were 100 and $140 \mathrm{~nm}$, respectively). All the scale bars in (a) are $200 \mathrm{~nm}$. (c) In situ extinction spectra of Au lattice structures polarized at $0.8 \mathrm{~V}$ vs. $\mathrm{Ag} / \mathrm{AgCl}$ for $600 \mathrm{~s}$ in $0.1 \mathrm{M} \mathrm{NaClO}_{4}$ containing $10 \mathrm{mmol} \mathrm{dm}^{-3} \mathrm{KBr}$. The values of $a$, diameter of each particle, and structure height are 400,140, and $100 \mathrm{~nm}$, respectively. (d) Plots of the (upper) maximum resonance wavelength and (bottom) FWHM of the extinction spectra in (c) as a function of the electrochemical potential polarization time. Reprinted with permission from Ref. 41

SLR mode was observed as a function of the potential applying time (Fig. 3c). This optical property change was derived from the changes in not only the size of each particle but also the lattice spacing caused by the electrochemical oxidation reactions. This is because that the SLR property is sensitively dependent on both the particle size and the $a$ value. For example, the $100 \mathrm{~nm}$ increase of either $a$ or the diameter of each particle leads to the $100 \mathrm{~nm}$ red shift of SLR wavelength. ${ }^{46}$ Importantly, at the specific polarization time ( $=\sim 120 \mathrm{sec})$, we have observed that the line width of the spectrum (FWHM) achieved a minimum. From AFM measurements, it was found that the diameter and structure height were homogeneously decreased to $80 \%$ of the initial vales. By this dissolution, the initial FWHM value of $0.165 \mathrm{eV}$ at $730 \mathrm{~nm}$ decreased to the minimum value of $0.145 \mathrm{eV}$ at $714 \mathrm{~nm}$. The line width of the plasmon mode corresponds to the lifetime of the SLR mode. From the current decrease of FWHM value $(0.02 \mathrm{eV})$, it can be estimated as the 1.5 times $(\sim 10 \mathrm{fs})$ increase of SLR lifetime. Therefore, the results in

Fig. 3d indicate that our approach can be applied for an improvement in the lifetime of the SLR mode estimated to up to the order of $100 \mathrm{fs}$, which is difficult to achieve using the conventional lithography method.

Through all these attempts, it has been successfully demonstrated that our established electrochemical structure control method can achieve the ultimate energy confinement and extension of the lifetime of light for efficient electrochemical reaction fields.

\section{Probing the Unique Molecular Process at the Plasmonic Field}

A spectroscopy method that can be used to probe the molecular behavior and the light-absorption process with high sensitivity is necessary for understanding the light-matter interaction at the interface. Since the enhancement of Raman scattering at the nanostructured metal surface was first observed in $1974,{ }^{47}$ the SERS measurement has been considered as a powerful tool to detect the small number of molecules at the nanoscale interface. ${ }^{48}$ Recent studies have demonstrated even single-molecule detection using well-defined nanostructures. ${ }^{49,50}$ SERS enhancement factors ranging between $10^{5}$ and $10^{15}$ have been experimentally obtained. ${ }^{51}$ Electromagnetic (EM) and charge-transfer (CT) mechanisms have been proposed as mechanisms for the Raman scattering enhancement. ${ }^{52,53}$ The EM mechanism originates from the strong electromagnetic field of LSPR with an enhancement factor of $\sim 10^{10}$ achieved. ${ }^{54}$ Considering the difference between the experimentally observed SERS enhancement factors and the limit for the EM mechanism, the CT mechanism, which is the resonance with the incident light between the molecular orbital and the Fermi level of the metal, contributes to the enhancement. ${ }^{55}$ Because of this surface dependent process, the unique molecular selectivity properties of SERS can be explained. Thus, the control of the resonant electronic excitation of molecules is an important factor for SERS measurements. Taking the resonance effect into account, the electrochemical potential tuning is effective for SERS measurements for the precisely tuned electronic structure of the interfaces. ${ }^{55-57}$

To understand the molecular behavior strongly interacted with the plasmonic field at the well-defined nanostructure surface, we attempted to combine SERS measurements with the electrochemical potential control method. ${ }^{58-62}$ As mentioned above, the electrochemical potential scan can easily change not only the molecular orientation via the surface charge but also the resonance effect. As one example, we have conducted electrochemical SERS measurements using bi-analyte solution containing 4'4-bipyride and 2'2bipyride. It was found that the surface absorption species can be successively changed by the potential scan (Fig. 4a). As can be found from Fig. $4 \mathrm{~b}$, the dominant adsorbate was switched by the potential scan with the superior reversibility. The plots in Fig. 4c indicate the correlation plots prepared from Fig. 4a. The solid and broken lines in the figures correspond to the negative and positive potentials, respectively. The linear tendency in such correlation plots implies the stable adsorption with the well-defined orientation against the surface. Importantly, we have confirmed the formation of a unique molecular condensation state, especially in the negative potential region, which can allow the CT resonance condition of the target molecules (Fig. 4c). This can be found from the scattering of the plots, corresponding to the fluctuation of the orientation in the condensation phase of molecules. The fact that the molecular surface state is affected under the CT condition would imply that the electronic resonance effect at the well-controlled nanostructure surface could modify the chemical reactivity of the system.

Under the formation of the strong coupling states between dye exciton and LSPs, the absorption and emission properties of the system can be drastically changed. In addition, the lifetime of the excited state and the chemical reactivity can be modulated. The 
(a)

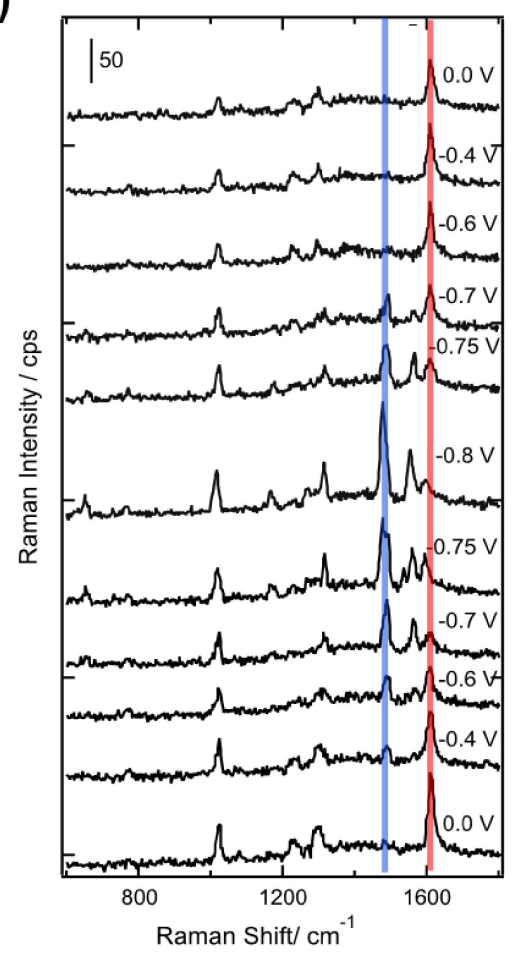

(b)

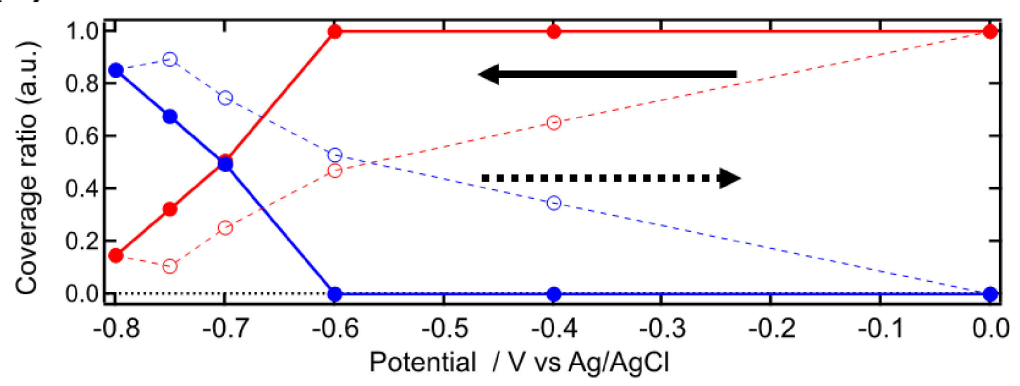

(c)

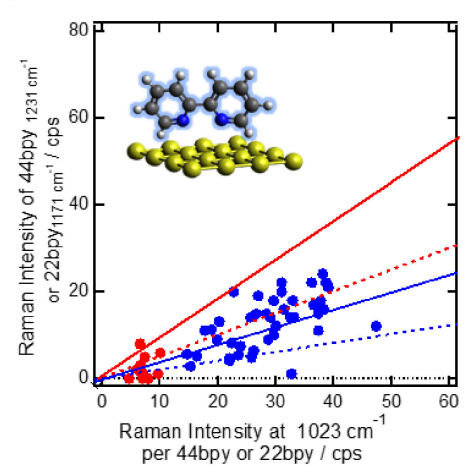

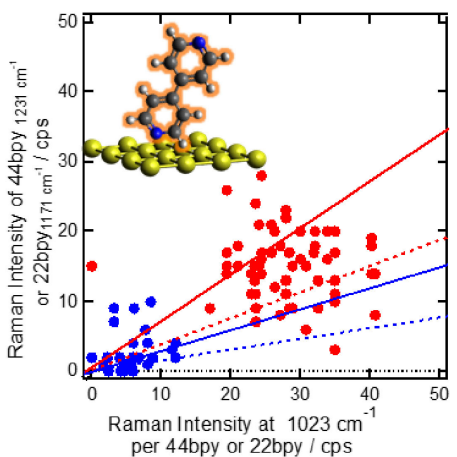

Figure 4. (a) Electrochemical SERS spectra obtained in the mixed solution of $1 \mathrm{mmol} \mathrm{dm}^{-3} 44 \mathrm{bpy}$ and $22 \mathrm{bpy}$ in aqueous $0.1 \mathrm{~mol} \mathrm{dm}^{-3}$ $\mathrm{NaClO}_{4}$. (b) Relative ratios of each Raman band for (red) 44 bpy and (blue) 22 bpy. The solid and broken arrows indicate the direction of the potential scan. (c) Correlation plots of SERS intensity for (red) 44 bpy and (blue) 22 bpy collected at (left) 0 and (right) $-0.8 \mathrm{~V} \mathrm{vs.} \mathrm{Ag} / \mathrm{AgCl}$, respectively. Reprinted with permission from Ref. 61.

formation of the strong coupling states also affects significantly on the resonance process at Raman scattering, leading to the enhancement of SERS intensity as the function of the coupling strength. ${ }^{63}$ From these facts, it is apparent that, in addition to the control of the strong coupling regime, probing the molecules under the strong coupling condition is an important issue. As mentioned above, the LSPR property can be tuned by the electrochemical potential scan, which enables the electron density of the system to be changed. ${ }^{64}$ We have thus attempted to actively tune the coupling strength by tuning the electrochemical potential of metal nanostructures. ${ }^{65-67}$ As a strong coupling system, we have deposited dye molecules onto the plasmonic structures (Fig. 5a). The extinction spectra of the plasmonic structured covered with and without dye molecules are shown in Fig. 5b. The appearance of peak splitting indicates the formation of the strong coupling regime. The width of the splitting corresponds to the coupling strength. Interestingly, this coupling strength showed parabolic behavior depending on the electrochemical potential (Figs. $5 \mathrm{c}$ and $5 \mathrm{~d}$ ). In the negative potential region, the decrease of the coupling strength can be considered as reduction reactions of the molecules, resulting in a change in the optical property of the molecule. However, only from the extinction spectra, it was difficult to understand the origin of the change in the coupling strength in the positive region.

We then performed electrochemical SERS measurements to clarify the origin of that behavior (Fig. 6a). By analyzing the Raman intensity ratios between the molecular vibration mode and fluorescence (= background of the spectrum), the distance between molecules and the metal surface can be estimated. Through the analyses (Figs. $6 \mathrm{~b}$ and $6 \mathrm{c}$ ), we clarified that the distance between the metal and molecules gradually changed depending on the electrochemical potential, as illustrated in Fig. 6d. The minimum distance led to the highest coupling strength. From these measurements, we successfully achieved electrochemical control of the strong coupling regime, leading to the efficient resonant effect, based on molecular manipulation at the nanoscale electrified interface. Recently, we have attempted to couple the dye molecules with the lattice structure because, owing to the relatively longer lifetime of the SLR mode, a more efficient and broader range of the coupling field can be expected. As a result, unique electrogenerated chemiluminescence was observed depending on the SLR property. ${ }^{68}$

Through those attempts, we have successfully functionalized the nanoscale interface via the light-matter interaction, leading to the efficient resonance effect between the molecules and nanostructured electrode.

\section{Clarification of Electron Transfer Process on Plasmonic Photoconversion Electrode}

During the non-radiative decay of LSPR, the electron-hole pair is generated, which can be used in the chemical reactions. ${ }^{69-71}$ Regarding to the electron-hole excitation, this process is induced through intraband and/or interband transitions via Landau damping; then, the excited electrons are successively multiplied by the electron-electron scattering. The timescales for the former and latter reach up to ten and a few hundred femtoseconds, respectively. Usually, the recombination process is much faster than the electron transfer; however, by supporting the plasmonic nanostructures on the semiconductor, the charge separation efficiency can be improved. It is also important that the visible-light response ability can be added to wide-band semiconductors, which cannot absorb the visible light, such as $\mathrm{TiO}_{2}$. To the best of our knowledge, the first experimental demonstration of photocurrent generation on $\mathrm{n}-\mathrm{TiO}_{2}$ under visible-light illuminations was reported by Yoko et al. in 1996. ${ }^{72}$ In 2005, Tian and Tatsuma proposed the mechanism for this system. ${ }^{73}$ According to their mechanism, the electrons excited within the plasmonic nanostructure are injected into the conduction 
(a)
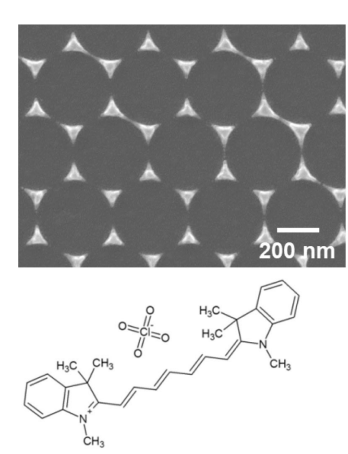

Energy / eV
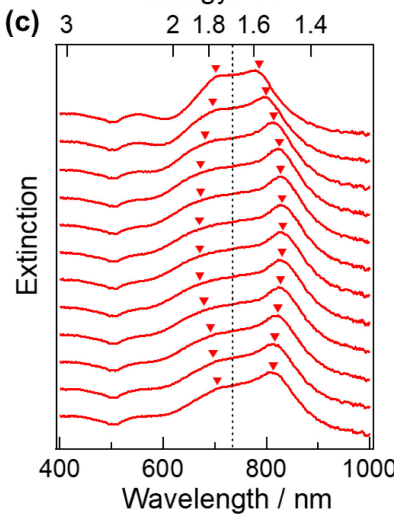

Figure 5. (a) SEM image of $\mathrm{Au}$ plasmonic structures and chemical structure of dye molecule (HITC). (b) Extinction spectra for plasmonic nanostructure (black) with and (gray) without dye molecule coverage. (c) Electrochemical in situ extinction spectra of plasmonic nanostructures covered with dye molecular layer. The electrochemical potential was scanned from 0.3 to $-0.8 \mathrm{~V}$ vs. $\mathrm{Ag}$ / $\mathrm{AgCl}$, then rescanned to $0.3 \mathrm{~V}$, corresponding to top to bottom. The vertical broken lines in (b) and (c) correspond to the absorption peak of HITC molecules. (d) Energy splitting value for each electrochemical potential estimated from (c). Reprinted with permission from Ref. 65.

band of $\mathrm{n}-\mathrm{TiO}_{2}$ and remained holes are consumed through the oxidation reactions at the metal-semiconductor interface. Recent studies have also proposed other mechanisms, such as the direct excitation from the metal to semiconductor or plasmon-induced metal-to-semiconductor interfacial electronic transfer transition. ${ }^{74}$ This system is recognized as the plasmonic photoconversion system and has been applied for various reaction systems, even for the water-splitting reaction. ${ }^{75}$ To establish high-efficiency plasmonbased photovoltaic devices, clarification of not only the electron transfer process but also the electrochemical potential of the reactive species are important issues.

We have attempted to obtain such information through electrochemical SERS measurements using Au nanostructures supported on $\mathrm{n}-\mathrm{TiO}_{2}$ electrodes. ${ }^{76-79}$ For visualization of the reaction active sites, we performed the photo-induced oxidation reaction of a conductive polymer (polypyrrole). The well-defined plasmonic structures were prepared using electron-beam lithography, as shown in the SEM images (Fig. 7a). This bow-tie structure shows different spatial distributions of the electric field depending on the polarization direction of the incident light. The Raman measurements were performed using a $\lambda_{\mathrm{ex}}=785 \mathrm{~nm}$ laser polarized along the longand short-axis directions, as shown in the figure. Interestingly, the spatial distributions of the deposition positions were dependent on the polarized direction of incident light. According to previous theoretical efforts, these deposition positions correspond to the spatial distribution of the plasmonic field. This means that the plasmon-induced photoconversion process is preferably triggered at the plasmonic field. We thus succeeded in visualization of the spatial distribution of the reaction active sites on the plasmon-induced photoconversion system.

The deposition of the polypyrrole was confirmed by electrochemical Raman measurements (upper column of Fig. 7b). The interesting point is that the deposition behavior showed an electrochemical potential dependence (bottom column of Fig. 7b). At more positive potential than the flat-band potential of $n-\mathrm{TiO}_{2}$, the polymer deposition was confirmed. Under the more negative polarization condition, no deposits were observed. Considering this finding and the incident photon energy $\left(\lambda_{\mathrm{ex}}=785 \mathrm{~nm}(1.58 \mathrm{eV})\right)$, the electron transfer process can be described as shown in Fig. 7c. In the positive potential region, the space charge layer forms at the metalsemiconductor interface, leading to efficient charge separation, whereas at more negative potential than the flat band potential $(-0.68 \mathrm{~V}$ vs. $\mathrm{Ag} / \mathrm{AgCl})$, the electrons and holes are easily recombined. One of the important facts in our proposed mechanism is that the holes are generated at more positive potential than the Fermi level of the $\mathrm{Au}$, and its electrochemical potential is estimated to be $\sim 0.9 \mathrm{~V}$ vs. $\mathrm{Ag} / \mathrm{AgCl}(=-0.68 \mathrm{~V}-1.58 \mathrm{eV})$. Taking the fast recombination of the excited electrons into account, it can be expected that the hole trapped site formed at the interface would contribute to the effective electron transfer reaction. Through our investigations, information on the absolute electrochemical potential of the generated reactive species was obtained for the first time.

In further investigations, we have applied this method for rod structures that can excite the higher order mode. Within the rod structure with relatively high aspect ratio, the propagation of the light leads to multipole modes (higher order, mode index number: $n=2,3,4$, and more) under the light polarized parallel to the long axis. We have prepared various rod structures with different aspect ratios and performed the polymerization of the pyrrole. Different spatial distributions of the deposits were observed depending on the rod length, as shown in Fig. 8a. In addition, we observed the periodic changes of the Raman intensity of deposits as a function of the rod length (upper part of Fig. 8b). Because only the odd modes can be excited under the perpendicular incident illumination, we can define each length in Fig. 8 as $n=1,3,5$, and 7, respectively. Estimation of the Raman enhancement factor for each rod length from the theoretical calculations (shown as the right axis of the upper column) indicated that the efficiency of the plasmon-induced oxidation reaction did not directly correlate with the electric field intensity. Furthermore, we successfully described the relationship between the rod length and the plasma and incident wavelength, as shown in the bottom column of Fig. 8. Therefore, we have achieved the reliable estimation of the reaction efficiency and resonance wavelength dependence on the higher order mode.

We have also conducted Raman measurements by introducing a graphene layer at the plasmonic photoconversion electrode surface $\left(\mathrm{Au} / \mathrm{TiO}_{2}\right)$. It is well known that the wavenumber of G-band for graphene is sensitively dependent on the Fermi level of the substrate. In other words, the Fermi level of the electrode can be obtained from the wavenumber of G-band. Using this character, we attempted to probe the Fermi-level shift of plasmonic nanostructures during water-oxidation reactions. Generally, photoconversion ability is strongly dependent on the electrode, as observed in Fig. 9a. By using these two electrodes which shows the different catalytic performance prepared by the similar procedure, the electrochemical SERS spectra of graphene supported on the $\mathrm{Au} / \mathrm{TiO}_{2}$ electrodes corresponding to Fig. 9 a were obtained. As a common tendency, a shift of wavenumber was observed at a more positive potential region than the flat band potential; however, the shift of the wavenumber was distinct for each electrode (Fig. 9b). The obvious wavenumber shift indicates the positive shift of the Fermi level. This 
(a)

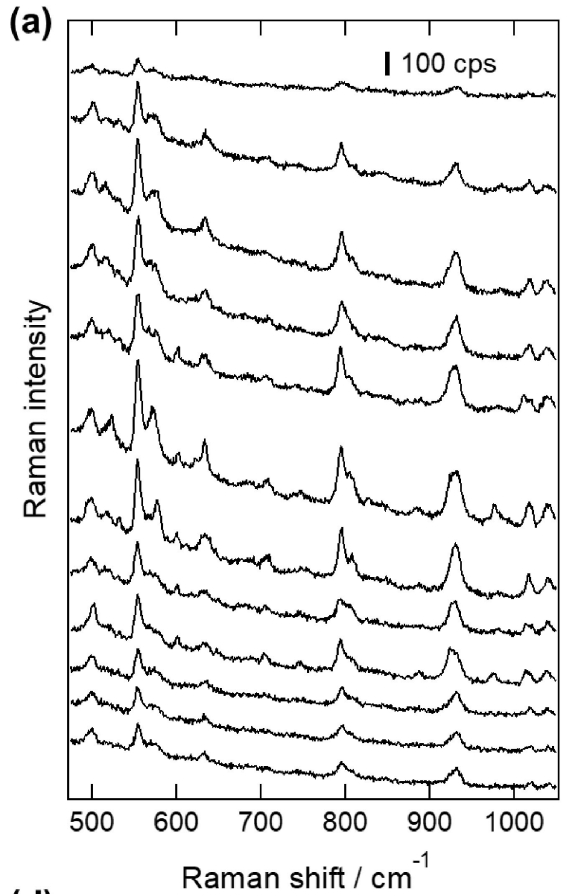

(b)

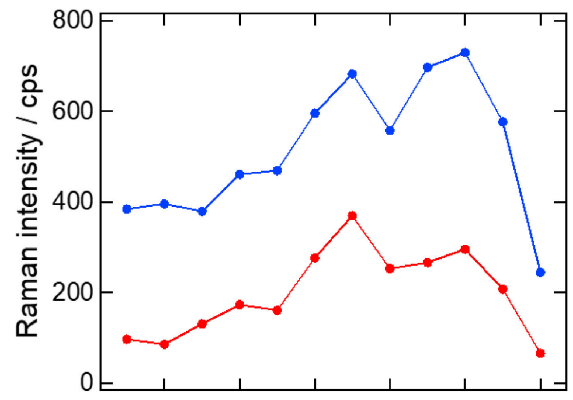

(c)

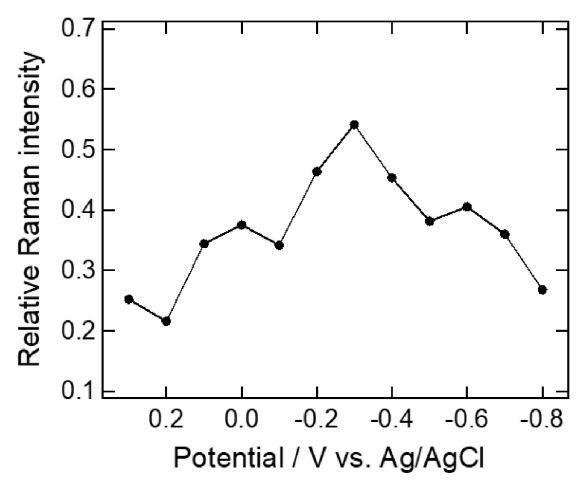

(d)
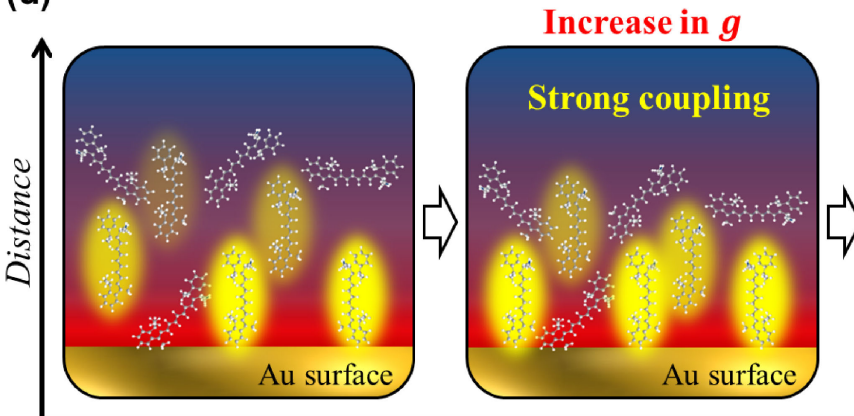

Electrochemical potential

Decrease in $g$

EC reduction

Positive

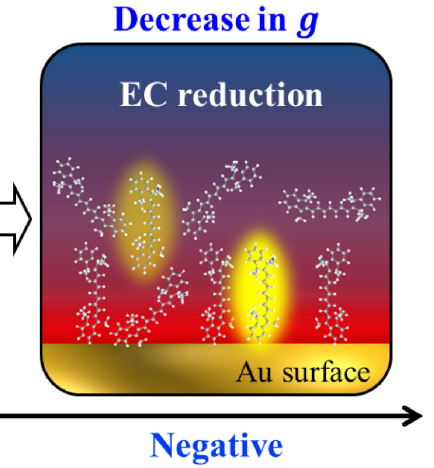

Figure 6. (a) Electrochemical SERS spectra of dye supported on plasmonic structure collected in $0.1 \mathrm{~mol} \mathrm{dm}^{-3} \mathrm{NaClO}_{4}$ aqueous solution. The electrochemical potential was changed from (bottom) 0.3 to (top) $-0.8 \mathrm{~V}$ vs. Ag/AgCl. (b) Raman band intensities at (red) 555 and (blue) $543 \mathrm{~cm}^{-1}$ and (c) the SERS intensity ratio at 555 and $543 \mathrm{~cm}^{-1}$ calculated from spectra in (a) as a function of the electrochemical potential. (d) Schematic illustration of the mechanism for change in coupling strength depending on the electrochemical potential. The vertical direction indicates the distance from the surface of the metal nanostructure. Reprinted with permission from Ref. 65.
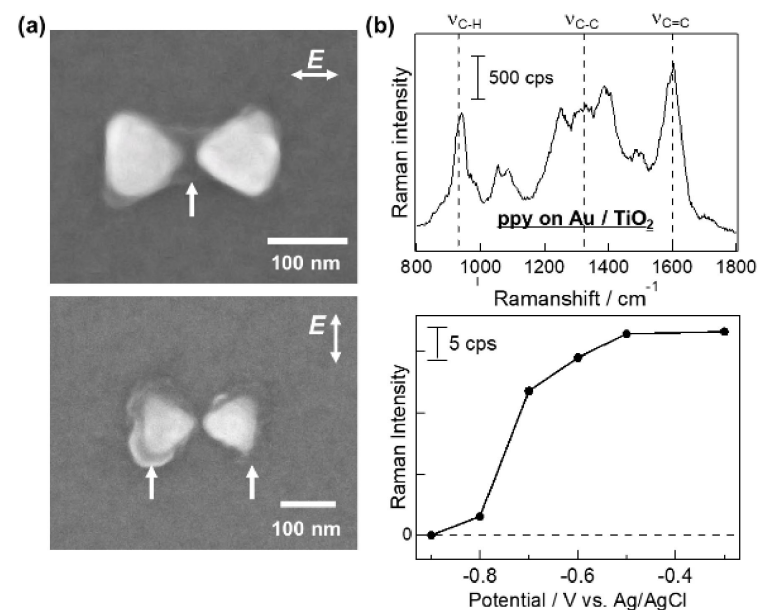

(c)

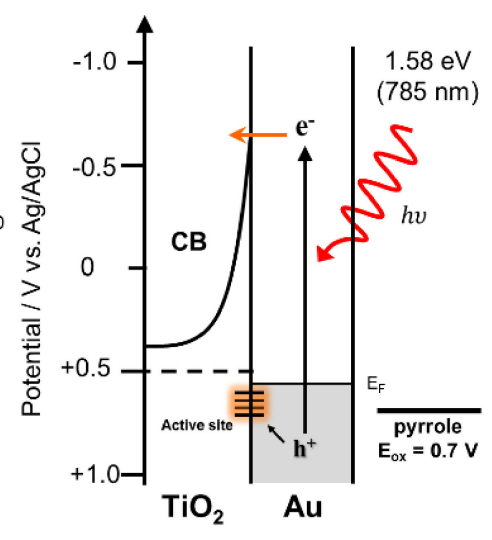

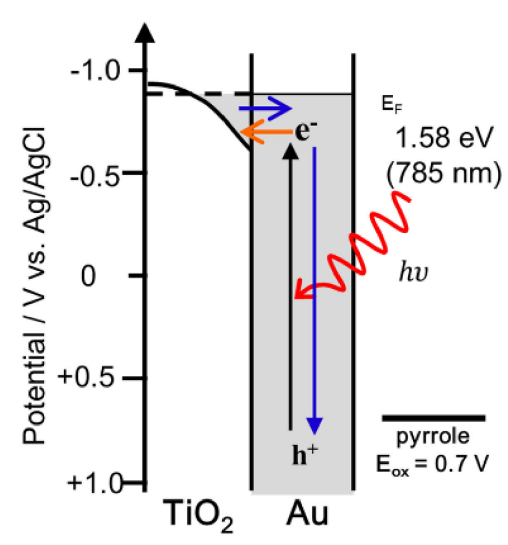

Figure 7. (a) SEM images of bow-tie structures after illumination in $1 \mathrm{mmol} \mathrm{dm}{ }^{-3}$ pyrrole $+0.1 \mathrm{~mol} \mathrm{dm}^{-3} \mathrm{NaClO}_{4}$ acetonitrile solution. The applied electrode potential was $0.3 \mathrm{~V}$ vs. $\mathrm{Ag} / \mathrm{Ag}^{+}$. The incident laser wavelength and light intensity were $785 \mathrm{~nm}$ and $0.1 \mathrm{~mW}$, respectively. The single-headed and double-headed arrows in each image indicate the deposited polymer and polarization direction of incident light, respectively. (b) Raman spectra of deposit and electrochemical potential dependence of the Raman intensity. (c) Energy diagrams of Au/ $\mathrm{TiO}_{2}$ electrodes at applied electrode potentials of (left) 0.5 and (right) $-0.9 \mathrm{~V}$ vs. $\mathrm{Ag} / \mathrm{AgCl}$. Reprinted with permission from Refs. 74 and 77. 
(a)
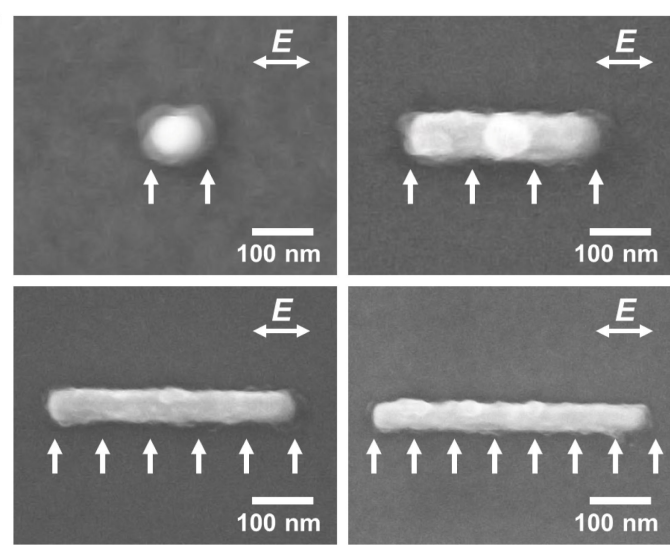

(b)

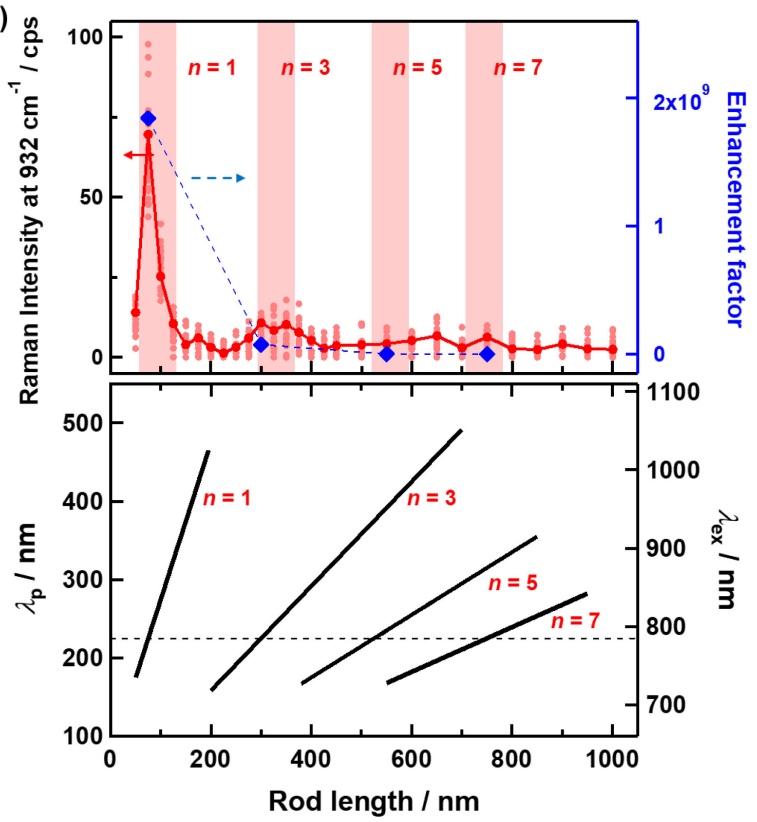

Figure 8. SEM images of $\mathrm{Au}$ nanorods after irradiation with $0.1 \mathrm{~mW}$ of $785-\mathrm{nm}$ laser. The rod lengths and irradiation times were (upper left) $75 \mathrm{~nm}, 5 \mathrm{~s}$; (upper right) $300 \mathrm{~nm}, 10 \mathrm{~s}$; (bottom left) $550 \mathrm{~nm}, 10 \mathrm{~s}$; and (bottom right) $750 \mathrm{~nm}, 10 \mathrm{~s}$. Laser irradiation was conducted in $1 \mathrm{mmol} \mathrm{dm}^{-3}$ pyrrole $+0.1 \mathrm{~mol} \mathrm{dm}^{-3} \mathrm{NaClO}_{4}$ acetonitrile solution at a static potential of $0.3 \mathrm{~V}$ vs. $\mathrm{Ag} / \mathrm{Ag}^{+}$. The doubleheaded arrows shown in each image indicate the polarization direction of incident light. (b) Plots of (left axis) Raman intensity and (right axis) the enhancement factor of Raman intensity estimated from FDTD calculations. The plasmon mode index estimated from (a) is indicated as the colored region. (bottom) Correlation between plasma and incident-light wavelength for each mode index as a function of rod length. Reprinted with permission from Ref. 77.

character can be understood as the energy diagram depicted in Fig. 9c. As mentioned above, the hole trapping sites play an important role. In the case for the electrode with higher performance, the large number of the hole trapping sites leads to the effective consumption of holes, resulting in the relatively negative Fermi level. Contrary to this, in the case for less trapping sites (lower activity), the Fermi level of Au shifts to much positive potential region due to the accumulation of holes. This proposed mechanism is supported by the examination of the incident light intensity dependence, leading to the change in the number of excited holes and electrons. ${ }^{76}$ As the results, the excess holes under the intense light intensity led to the positive Fermi level shift. Therefore, we have succeeded in monitoring the relationship between the number of hole trapping sites and the different Fermi levels, resulting in different catalytic activity. Thus, our present graphene-based Raman measurements allow us to prove the activity of the plasmonic photoconversion electrode. From all the Raman measurements, we successfully proposed the detailed electron transfer process and the absolute electrochemical potential of the reaction active species and its Fermi level. Based on the above information, we recently attempted to establish a plasmonic cathode electrode that can trigger a visible-light-driven hydrogen evolution system by combining the plasmonic nanostructures with a p-type semiconductor electrode. ${ }^{80}$ To date, we have achieved hydrogen-evolution reactions under visible-light illumination. Interestingly, our plasmonic cathode electrode exhibited unique $\mathrm{pH}$ dependence. Consequently, we were able to achieve unique properties of the photoelectrochemical reactions at the well-controlled nanostructure interface.

\section{Investigation of Unique Reaction Selectivity on Nanostructured Electrode}

At the functionalized nanostructured interface, unique reaction selectivity was observed. We have investigated the hydrogen evolution reactions (HER) at the well-controlled nanostructured interface. The HER is one of the well-investigated reactions in the electrochemical field. ${ }^{81-83}$ It is well known that the surface molecular process involves complicated reactions. During the cathodic reaction of $\mathrm{H}_{2} \mathrm{O}$, the absorbed hydrogen atoms form (recognized as the Volmer step) followed by the desorption process (Heyrovsky step). The chemical desorption (Tafel) process is also triggered at the highly catalytic surface. Basically, the ratedetermining step of the HER is dependent on the surface metal species because the metal-hydrogen bond determines the surface process, known as the Sabatier principle. ${ }^{84,85}$ Thus, there is a limitation for the modulation of the surface process at the conventional metal electrode. As our first approach, we monitored the molecular catalysis (4'4-bipyridine) for the electrochemical HER at each electrochemical potential through electrochemical SERS measurements. ${ }^{86}$ At negative potential $(-0.9 \mathrm{~V}$ vs. $\mathrm{Ag} / \mathrm{AgCl})$, the appearance of the normally forbidden mode was observed, indicating the specific electronic interaction exhibiting the CT process (Fig. 10). This result indicates that the specific interaction can accelerate the catalytic activity at the nanostructured surface.

To examine the unique reaction selectivity on the nanostructured electrodes, we recently investigated the isotopic effect on the $\mathrm{Ag}$ nanostructured surface. Because the isotopic HER is a complicated process, as shown in Fig. 11a, tuning of the isotopic selectivity is one of the great challenges. The isotopic selectivity of the HER is directly correlated with the rate-determining step; therefore, investigation of the isotopic selectivity can provide insight into the surface process for the HER. ${ }^{87-91} \mathrm{We}$ have established an electrochemical mass spectroscopy system to detect the reaction products in real time to monitor the isotopic selectivity (Fig. 11b). ${ }^{92}$ From the mass spectrum obtained under the static negative potential polarization condition, which triggers the HER, we have calculated the separation factor $\left(S_{\mathrm{D}}: S_{\mathrm{D}}=\{\mathrm{H} / \mathrm{D}\}_{\text {gas }} /\{\mathrm{H} / \mathrm{D}\}_{\text {liquid }}\right)$ to discuss the reaction characteristics (Fig. 11a). In the experiments, we estimated the $S_{\mathrm{D}}$ values on smooth Pt and $\mathrm{Ag}$ electrodes. Nanostructured Pt and $\mathrm{Ag}$ electrodes were also prepared using the electrochemical method. As shown in Fig. 11, although there were no distinct changes for either the smooth or nanostructured Pt electrodes, drastic improvement of the $S_{\mathrm{D}}$ value was confirmed for the nanostructured $\mathrm{Ag}$ electrodes, indicating the acceleration of the $\mathrm{H}_{2}$ generation (Figs. 11c and 11d). This finding originates from the selective acceleration of the surface processes, such as the Volmer or Tafel processes, at the nanostructured Ag surface. Because these processes can be improved at the highly catalytic surface, this result indicates that, by controlling the nanoscale interface structure, 
Electrochemistry, (in press) 1-11

(a)

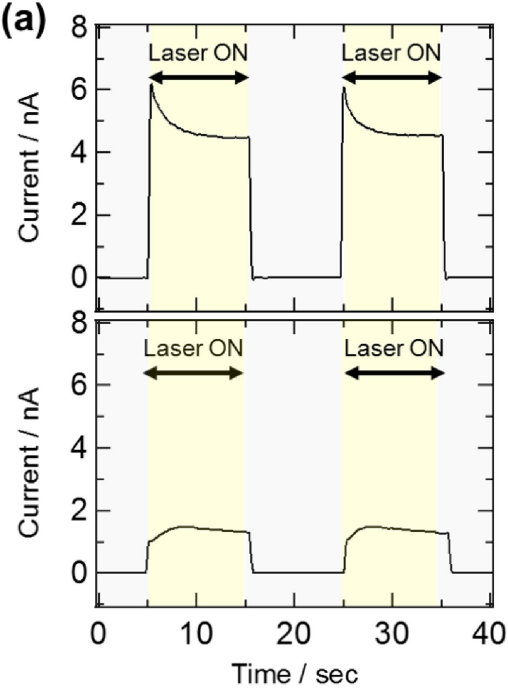

(b)

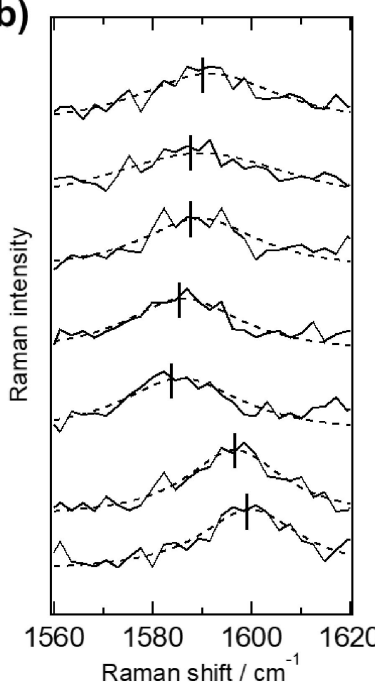

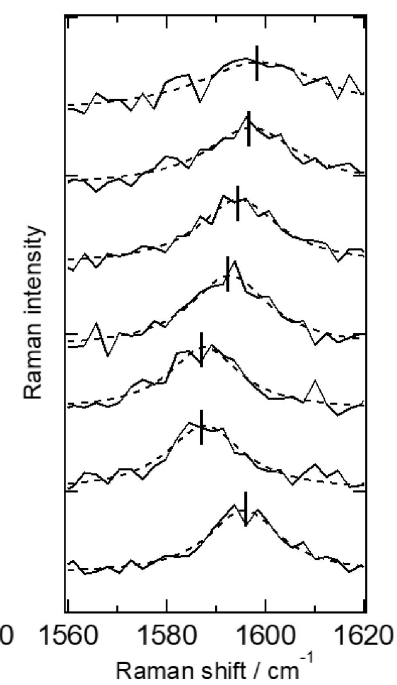

(c)
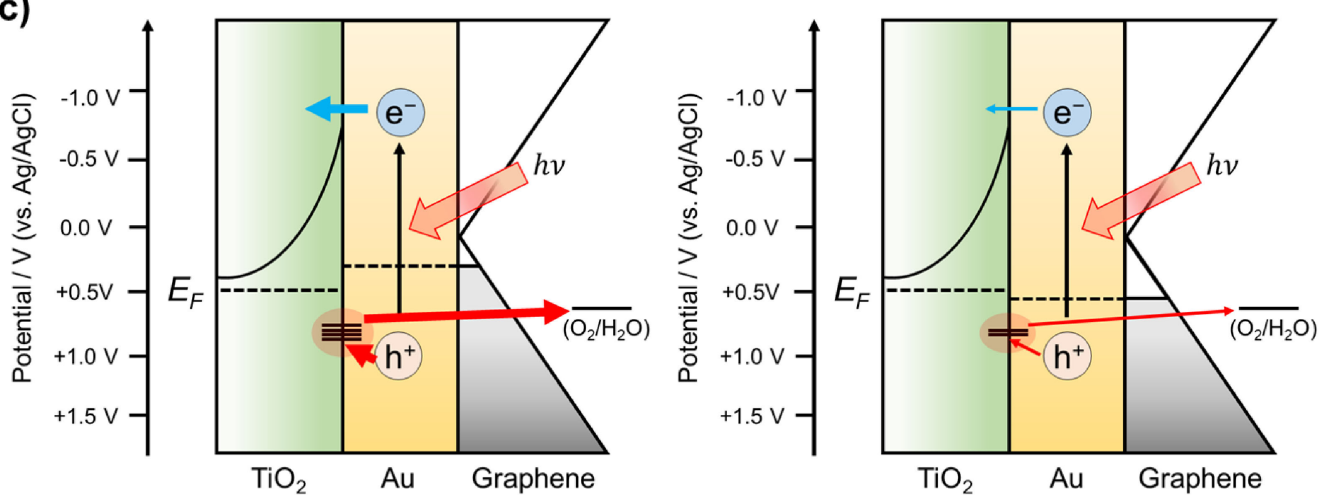

Figure 9. (a) Photocurrent responses of different $\mathrm{Au}$ nanostructure-supported $\mathrm{n}-\mathrm{TiO}_{2}$ electrodes. The photocurrents were obtained under illumination of a $785-\mathrm{nm}$ laser with $1.0 \mathrm{~mW}$. The electrochemical potential was set to $0.5 \mathrm{~V} \mathrm{vs}$. $\mathrm{Ag} / \mathrm{AgCl}$. (b) Electrochemical Raman spectra of plasmonic electrodes corresponding to the (left) upper and (right) bottom panels in (a). The electrochemical potential was scanned from -0.9 to $0.9 \mathrm{~V}$ vs. $\mathrm{Ag} / \mathrm{AgCl}$ at an interval of $0.3 \mathrm{~V}$, corresponding to the bottom to top. (c) Schematic energy diagrams for each electrode for (a) and (b). Reprinted with permission from Ref. 76.

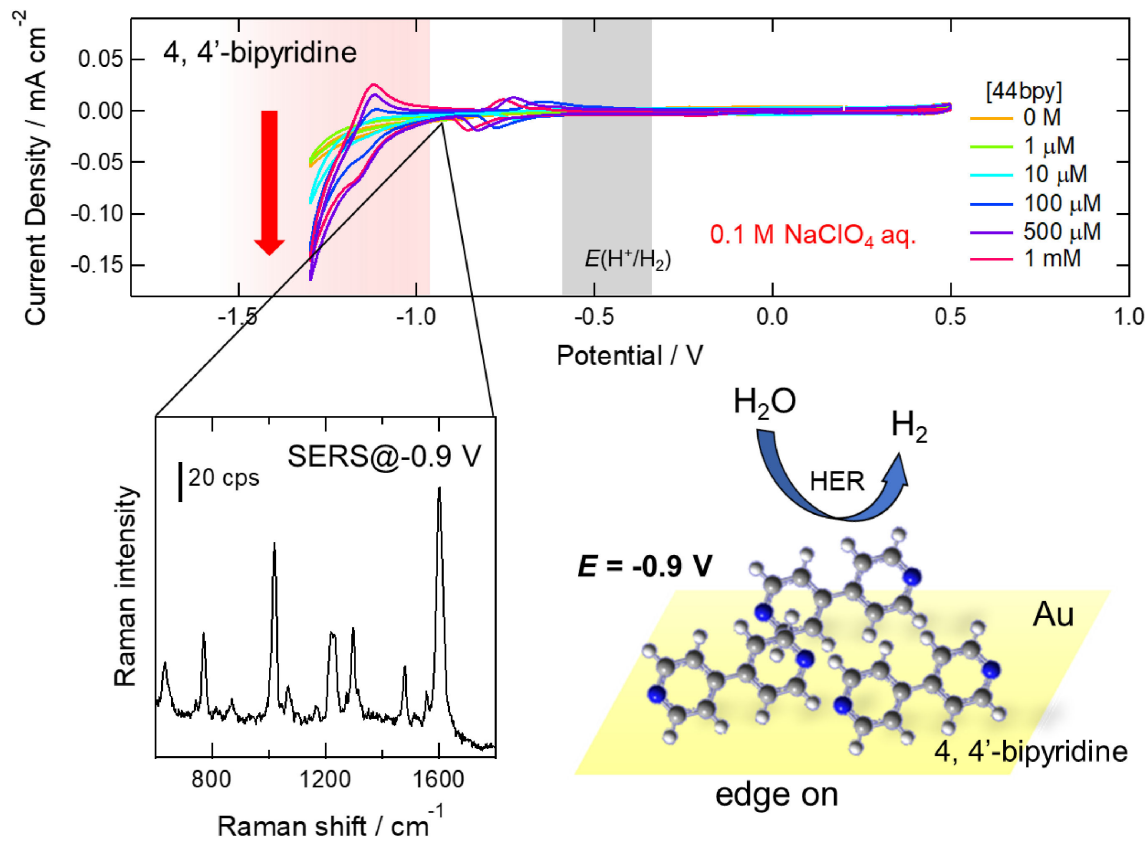

Figure 10. Cyclic voltammogram of $\mathrm{Au}$ electrode immersed in aqueous $0.1 \mathrm{moldm}^{-3} \mathrm{NaClO}_{4}$ with $1 \mathrm{mmol} \mathrm{dm}^{-3} 44 \mathrm{bpy}$ and electrochemical SERS spectrum of 44 bpy obtained at $-0.9 \mathrm{~V}$ vs. $\mathrm{Ag} / \mathrm{AgCl}$. Schematic of molecular catalytic process of 44 bpy. Reprinted with permission from Ref. 84. 
(a)
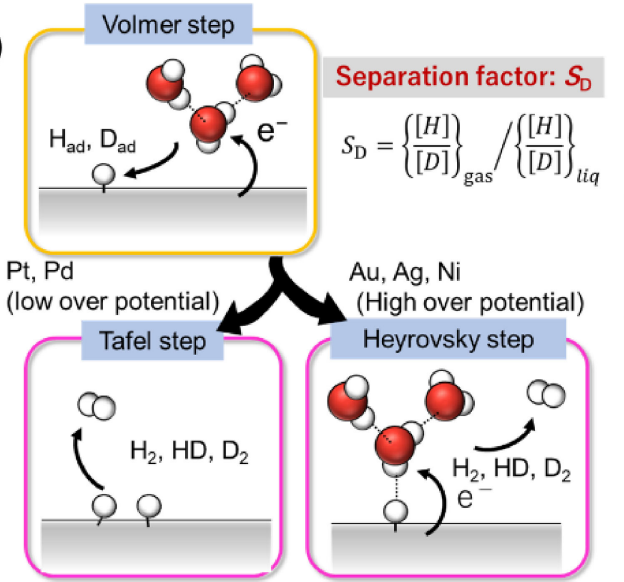

(c)

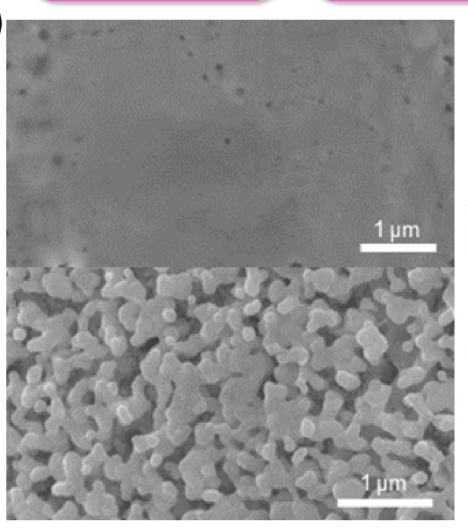

Separation factor: $S_{0}$ $S_{\mathrm{D}}=\left\{\frac{[H]}{[D]}\right\}_{\text {gas }} /\left\{\left[\frac{[H]}{[D]}\right\}_{l i q}\right.$

$\mathrm{Au}, \mathrm{Ag}, \mathrm{Ni}$ Heyrovsky step

(b)

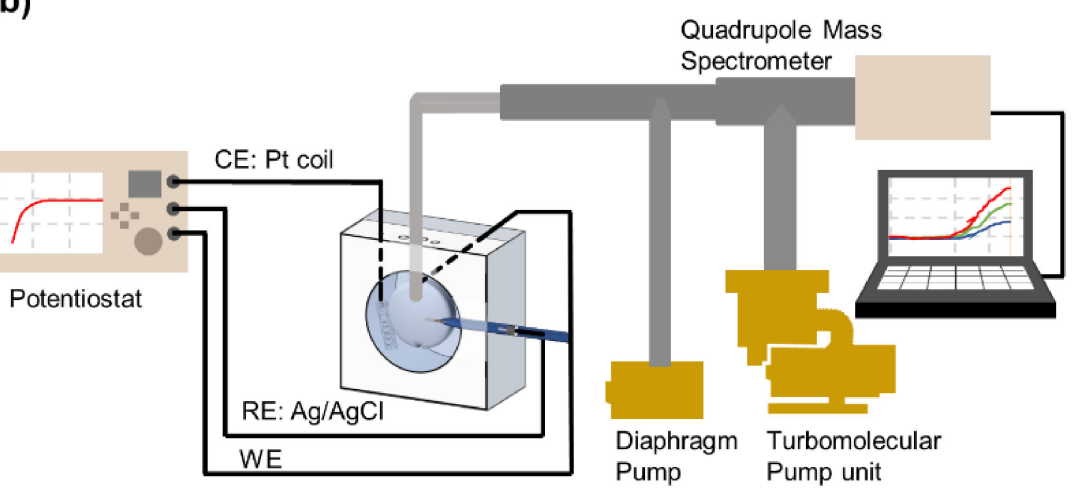

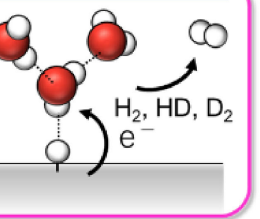

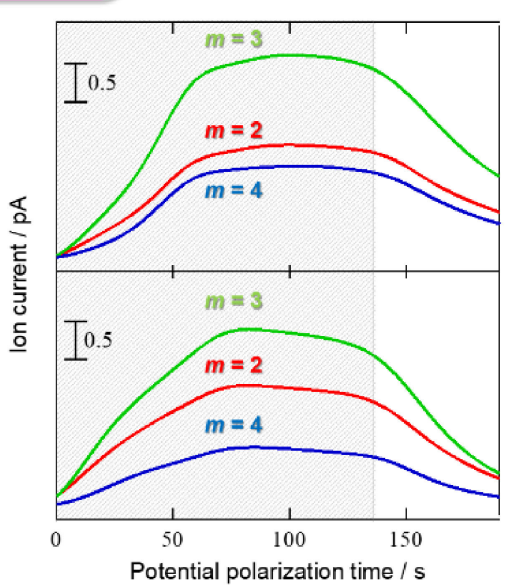

(d)

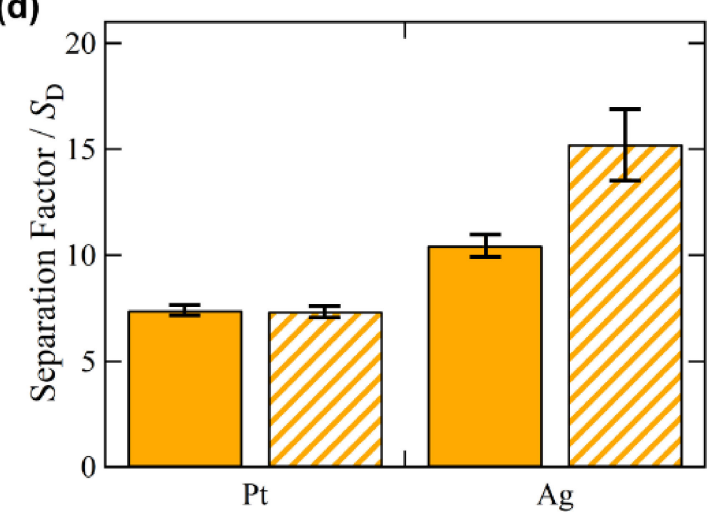

Figure 11. Schematic illustrations of (a) isotopic HER process and (b) our electrochemical mass spectroscopy system. (c) (left) SEM images of (upper) smooth and (bottom) nanostructured Ag electrode. (right) Electrochemical mass spectra obtained with (upper) smooth and (bottom) nanostructured $\mathrm{Ag}$ electrodes in mixed solution of $\mathrm{H}_{2} \mathrm{O}$ and $\mathrm{D}_{2} \mathrm{O}$. The mixed ratio of $\mathrm{H}_{2} \mathrm{O}$ and $\mathrm{D}_{2} \mathrm{O}$ was 1 to 10. The applied electrode potential was -1.4 vs. RHE. (d) Separation factors for smooth and nanostructured Pt and Ag electrodes estimated from Fig. 11c. Reprinted with permission from Ref. 90.

specific catalytic sites can be obtained on the non-catalytic metal surface. Through all the works described in this review, we have successfully demonstrated that the control and understanding of the nanoscale interface would provide a breakthrough for efficient electrochemical reactions beyond the limitation of the system.

\section{Conclusions}

In this review, we summarized recent progress to understand and control the electrochemical and photoelectrochemical reactions at the nanoscale interface via control of the metal nanostructures and electrochemical spectroscopic measurements. We have successfully proposed various interesting molecular behaviors and the detailed electron transfer process at the nanostructured interface. This insight suggests the possibility of the realization of highly effective electrochemical reactions through the precise control of the electrified interface. Based on these findings, we have attempted to investigate the unique reaction selectivity of the electrochemical reactions, which is derived from the functionalization of the nanoscale interface. We believe that our insight could open a path for the realization of ultimate high-efficiency electrochemical reactions.

\section{Acknowledgments}

Several of the works described in this review were financially supported by Grants-in-Aid for Scientific Research (Nos. 16K17848, JP16H06506, JP18H05205, and JP20H05084) from the Ministry of Education, Culture, Sports, Science, and Technology of Japan. Support from the Photo-excitonix Project in Hokkaido University is also acknowledged. The authors are grateful to all collaborators and members of the Physical Chemistry Laboratory at Hokkaido University for fruitful discussion and support.

\section{References}

1. Z. W. Seh, J. Kibsgaard, C. F. Dickens, I. Chorkendorff, J. K. Nørskov, and T. F. Jaramillo, Science, 355, eaad4998 (2017).

2. H.-J. Yin, J.-H. Zhou, and Y.-W. Zhang, Inorg. Chem. Front., 6, 2582 (2019).

3. Z. Zhou, Z. Huang, D. Chen, Q. Wang, N. Tian, and S. Sun, Angew. Chem., Int. Ed., 49, 411 (2010).

4. K. Ding, A. Gulec, A. M. Johnson, N. M. Schweitzer, G. D. Stucky, L. D. Marks, and P. C. Stair, Science, 350, 189 (2015).

5. M. I. J. Beale, N. G. Chew, M. J. Uren, A. G. Cullis, and J. D. Benjamin, Appl. Phys. Lett., 46, 86 (1985).

6. J.-F. Huang and Y.-C. Wu, ACS Sustain. Chem. Eng., 6, 8285 (2018).

7. C. J. Chen and R. M. Osgood, Phys. Rev. Lett., 50, 1705 (1983).

8. S. A. Maier and H. A. Atwater, J. Appl. Phys., 98, 011101 (2005).

9. K. A. Willets and R. P. Van Duyne, Annu. Rev. Phys. Chem., 58, 267 (2007)

10. J. A. Schuller, E. S. Barnard, W. Cai, Y. C. Jun, J. S. White, and M. L. Brongersma, Nat. Mater, 9, 193 (2010).

11. E. Hao and G. C. Schatz, J. Chem. Phys., 120, 357 (2004).

12. M. L. Brongersma, N. J. Halas, and P. Nordlander, Nat. Nanotechnol., 10, 25 (2015).

13. E. Kazuma, J. Jung, H. Ueba, M. Trenary, and Y. Kim, Science, 360, 521 (2018).

14. Y. Zhang, S. He, W. Guo, Y. Hu, J. Huang, J. R. Mulcahy, and W. D. Wei, Chem. Rev., 118, 2927 (2018).

15. E. Miliutina, O. Guselnikova, N. S. Soldatova, P. Bainova, R. Elashnikov, P. Fitl et al., J. Phys. Chem. Lett., 11, 5770 (2020).

16. K. Ueno, T. Oshikiri, Q. Sun, X. Shi, and H. Misawa, Chem. Rev, 118, 2955 
(2018)

17. D. L. Jeanmaire and R. P. Van Duyne, J. Electroanal. Chem. Interfacial Electrochem., 84, 1 (1977).

18. P. Anger, P. Bharadwaj, and L. Novotny, Phys. Rev. Lett., 96, 113002 (2006).

19. B. Sharma, R. R. Frontiera, A. I. Henry, E. Ringe, and R. P. Van Duyne, Mater. Today, 15, 16 (2012)

20. P. K. Jain, D. Ghosh, R. Baer, E. Rabani, and A. P. Alivisatos, Proc. Natl. Acad Sci. U.S.A., 109, 8016 (2012).

21. M. T. Trinh, M. Y. Sfeir, J. J. Choi, J. S. Owen, and X. Zhu, Nano Lett., 13, 6091 (2013).

22. M. Takase, H. Ajiki, Y. Mizumoto, K. Komeda, M. Nara, H. Nabika, S. Yasuda, H. Ishihara, and K. Murakoshi, Nat. Photonics, 7, 550 (2013).

23. J. A. Hutchison, T. Schwartz, C. Genet, E. Devaux, and T. W. Ebbesen, Angew. Chem., Int. Ed., 51, 1592 (2012).

24. A. E. Schlather, N. Large, A. S. Urban, P. Nordlander, and N. J. Halas, Nano Lett., 13, 3281 (2013)

25. R. Chikkaraddy, B. de Nijs, F. Benz, S. J. Barrow, O. A. Scherman, E. Rosta et al., Nature, 535, 127 (2016)

26. R. Quidant and C. Girard, Laser Photonics Rev, 2, 47 (2008).

27. M. L. Juan, M. Righini, and R. Quidant, Nat. Photonics, 5, 349 (2011)

28. W.-Y. Tsai, J.-S. Huang, and C.-B. Huang, Nano Lett., 14, 547 (2014).

29. S. Link and M. A. El-Sayed, J. Phys. Chem. B, 103, 8410 (1999).

30. L. Zhao, K. L. Kelly, and G. C. Schatz, J. Phys. Chem. B, 107, 7343 (2003).

31. I. Zorić, M. Zäch, B. Kasemo, and C. Langhammer, ACS Nano, 5, 2535 (2011).

32. V. Amendola, R. Pilot, M. Frasconi, O. M. Maragò, and M. A. Iatì, J. Phys. Condens. Matter, 29, 203002 (2017).

33. T. Atay, J. Song, and A. V. Nurmikko, Nano Lett., 4, 1627 (2004).

34. I. Romero, J. Aizpurua, G. W. Bryant, and F. J. García De Abajo, Opt. Express, 14 9988 (2006).

35. J. A. Scholl, A. García-Etxarri, A. L. Koh, and J. A. Dionne, Nano Lett., 13, 564 (2013).

36. F. Wen, Y. Zhang, S. Gottheim, N. S. King, Y. Zhang, P. Nordlander, and N. J. Halas, ACS Nano, 9, 6428 (2015).

37. R. Esteban, A. G. Borisov, P. Nordlander, and J. Aizpurua, Nat. Commun., 3, 825 (2012).

38. S. Oikawa, H. Minamimoto, and K. Murakoshi, Chem. Lett., 46, 1148 (2017).

39. S. Oikawa, H. Minamimoto, X. Li, and K. Murakoshi, Nanotechnology, 29, 045702 (2018)

40. S. Oikawa, H. Minamimoto, A. Ohnuki, and K. Murakoshi, Nanoscale, 12, 11593 (2020)

41. H. Minamimoto, S. Oikawa, T. Hayashi, A. Shibazaki, X. Li, and K. Murakoshi, J. Phys. Chem. C, 122, 14162 (2018).

42. E. M. Hicks, S. Zou, G. C. Schatz, K. G. Spears, R. P. Van Duyne, L. Gunnarsson, T. Rindzevicius, B. Kasemo, and M. Käll, Nano Lett., 5, 1065 (2005).

43. V. G. Kravets, F. Schedin, and A. N. Grigorenko, Phys. Rev. Lett., 101, 087403 (2008).

44. W. Zhou and T. W. Odom, Nat. Nanotechnol., 6, 423 (2011).

45. Y. Chu, E. Schonbrun, T. Yang, and K. B. Crozier, Appl. Phys. Lett., 93, 181108 (2008)

46. H. Minamimoto, S. Oikawa, T. Hayashi, A. Shibazaki, X. Li, and K. Murakoshi, J. Phys. Chem. C, 122, 14162 (2018).

47. M. Fleischmann, P. J. Hendra, and A. J. McQuillan, Chem. Phys. Lett., 26, 163 (1974).

48. P. L. Stiles, J. A. Dieringer, N. C. Shah, and R. P. Van Duyne, Annu. Rev. Anal Chem., 1, 601 (2008).

49. J. A. Dieringer, R. B. Lettan, K. A. Scheidt, and R. P. Van Duyne, J. Am. Chem. Soc., 129, 16249 (2007).

50. E. C. Le Ru, E. Blackie, M. Meyer, and P. G. Etchegoin, J. Phys. Chem. C, 111 13794 (2007)

51. P. G. Etchegoin and E. C. Le Ru, Phys. Chem. Chem. Phys., 10, 6079 (2008).
52. J. A. Creighton, Surf. Sci., 124, 209 (1983).

53. H. Yamada, H. Nagata, K. Toba, and Y. Nakao, Surf. Sci., 182, 269 (1987)

54. A. M. Michaels and L. Brus, J. Phys. Chem. B, 104, 11965 (2000).

55. J. R. Lombardi, R. L. Birke, T. Lu, and J. Xu, J. Chem. Phys., 84, 4174 (1986).

56. D.-Y. Wu, J.-F. Li, B. Ren, and Z.-Q. Tian, Chem. Soc. Rev, 37, 1025 (2008)

57. H. Minamimoto and K. Murakoshi, Curr. Opin. Electrochem., 22, 186 (2020).

58. X. Li, H. Minamimoto, and K. Murakoshi, Spectrochim. Acta, Part A, 197, 244 (2018).

59. R. Zhou, S. Yasuda, H. Minamimoto, and K. Murakoshi, ACS Omega, 3, 2322 (2018).

60. J. Zhang, R. Zhou, H. Minamimoto, S. Yasuda, and K. Murakoshi, Nano Lett., 19, 7887 (2019)

61. N. Oyamada, H. Minamimoto, Y. Wakisaka, and K. Murakoshi, Chem. Lett., 48, 820 (2019).

62. N. Oyamada, H. Minamimoto, and K. Murakoshi, J. Phys. Chem. C, 123, 24740 (2019)

63. F. Nagasawa, M. Takase, and K. Murakoshi, J. Phys. Chem. Lett., 5, 14 (2014).

64. A. C. Templeton, J. J. Pietron, R. W. Murray, and P. Mulvaney, J. Phys. Chem. B, 104, $564(2000)$

65. H. Minamimoto, F. Kato, F. Nagasawa, M. Takase, and K. Murakoshi, Faraday Discuss., 205, 261 (2017).

66. F. Kato, H. Minamimoto, F. Nagasawa, Y. S. Yamamoto, T. Itoh, and K. Murakoshi, ACS Photonics, 5, 788 (2018).

67. H. Minamimoto, F. Kato, and K. Murakoshi, J. Raman Spectrosc., 52, 431 (2021).

68. T. S. Heiderscheit, S. Oikawa, S. Sanders, H. Minamimoto, E. K. Searles, C. F. Landes, K. Murakoshi, A. Manjavacas, and S. Link, J. Phys. Chem. Lett., 12, 2516 (2021).

69. S. Link and M. A. El-Sayed, J. Phys. Chem. B, 103, 4212 (1999).

70. C. Sönnichsen, T. Franzl, T. Wilk, G. von Plessen, J. Feldmann, O. Wilson, and P. Mulvaney, Phys. Rev. Lett., 88, 077402 (2002).

71. R. Sundararaman, P. Narang, A. S. Jermyn, W. A. Goddard, and H. A. Atwater, Nat. Commun., 5, 5788 (2014).

72. G. Zhao, H. Kozuka, and T. Yoko, Thin Solid Films, 277, 147 (1996).

73. Y. Tian and T. Tatsuma, J. Am. Chem. Soc., 127, 7632 (2005).

74. K. Wu, J. Chen, J. R. McBride, and T. Lian, Science, 349, 632 (2015).

75. S. Mubeen, J. Lee, N. Singh, S. Krämer, G. D. Stucky, and M. Moskovits, Nat. Nanotechnol., 8, 247 (2013)

76. H. Minamimoto, T. Toda, R. Futashima, X. Li, K. Suzuki, S. Yasuda, and K. Murakoshi, J. Phys. Chem. C, 120, 16051 (2016).

77. J. Zhang, H. Minamimoto, S. Oikawa, T. Toda, X. Li, and K. Murakoshi, Chem. Lett., 47, 953 (2018).

78. H. Minamimoto, K. Yasuda, R. Zhou, X. Li, S. Yasuda, and K. Murakoshi, J. Chem. Phys., 152, 124702 (2020).

79. H. Minamimoto, T. Toda, and K. Murakoshi, Nanoscale, 13, 1784 (2021).

80. D. Sato, H. Minamimoto, and K. Murakoshi, Chem. Lett., 49, 806 (2020).

81. J. O. Bockris and E. C. Potter, J. Electrochem. Soc., 99, 169 (1952).

82. B. E. Conway and J. O. Bockris, J. Chem. Phys., 26, 532 (1957).

83. Y. Zheng, Y. Jiao, M. Jaroniec, and S. Z. Qiao, Angew. Chem., Int. Ed., 54, 52 (2015)

84. S. Trasatti, J. Electroanal. Chem. Interfacial Electrochem., 39, 163 (1972).

85. J. K. Nørskov, T. Bligaard, A. Logadottir, J. R. Kitchin, J. G. Chen, S. Pandelov, and U. Stimming, J. Electrochem. Soc, 152, J23 (2005).

86. Y. Yonezawa, H. Minamimoto, F. Nagasawa, M. Takase, S. Yasuda, and K. Murakoshi, J. Electroanal. Chem., 800, 7 (2017).

87. B. E. Conway, Proc. R. Soc. London. Ser. A Math. Phys. Sci, 247, 400 (1958).

88. B. E. Conway, Proc. R. Soc. A Math. Phys. Eng. Sci., 256, 128 (1960).

89. M. Salomon and B. E. Conway, J. Phys. Chem., 68, 2009 (1964)

90. J. O. Bockris and S. Srinivasan, J. Electrochem. Soc., 111, 858 (1964)

91. L. I. Krishtalik, Electrochim. Acta, 46, 2949 (2001)

92. H. Minamimoto, R. Osaka, and K. Murakoshi, Electrochim. Acta, 304, 87 (2019). 